\title{
A SPECTROSCOPIC STUDY OF EPSILON AURIGAE*
}

\author{
MAMORU SAIT $\bar{O}$ \\ Department of Astronomy, Kyoto University, Kyoto, Japan \\ SHUSAKU KAWABATA \\ Kyoto Gakuen University, Kameoka, Kyoto, Japan \\ KEIICH SAIJO \\ National Science Museum, Ueno Park, Tokyo, Japan \\ and \\ HIDEO SATO \\ Tokyo Astronomical Observatory, Mitaka, Tokyo, Japan
}

(Received 2 August, 1983)

\begin{abstract}
Epsilon Aurigae has been observed during ingress and totality between 1982 and 1983 at Okayama. Analyses of profiles of $\mathbf{H} \alpha$ line and of radial velocities of neutral hydrogen and metals show that the secondary component consists of at least three parts in structure.
\end{abstract}

\section{Introduction}

An eclipsing binary Epsilon Aurigae has a period of $27.1 \mathrm{yr}$ and the eclipse is occurring between 1982 and 1984. For the previous eclipses many observations were made in optical wavelength regions. The observed results have derived various models of the structure and physical state of the invisible secondary component (Kuiper et al., 1937; Gaposchkin, 1954; Hack, 1959; Huang, 1965; Kopal, 1954, 1971; Wilson, 1971). Campaign Newsletters of Epsilon Aurigae eclipse being published by Drs Hopkins and Stencel have announced that the present eclipse continues to progress on schedule and that many astronomers have been observing the eclipse on ultraviolet and infrared wavelength regions as well as optical region. Observations of polarization are also being done. We can expect that nature of the secondary is unveiled by these observations.

This report is preliminary results obtained by the $188 \mathrm{~cm}$ reflector of Okayama Astrophysical Observatory for variations of $\mathrm{H} \alpha$ profile and radial velocities of atoms with phase around the second contact. The results may give a constraint for models of the secondary.

\section{Profile of $\mathbf{H} \alpha$ Line}

Figure 1 shows profiles of the $H \alpha$ line on spectrograms with a dispersion of $8.3 \AA$ per $\mathrm{mm}$. The profile obtained outside eclipse, Figure 1a, is characterized by a relatively

\footnotetext{
* Paper presented at the Lembang-Bamberg IAU Colloquium No. 80 on 'Double Stars: Physical Properties and Generic Relations', held at Bandung, Indonesia, 3-7 June, 1983.
} 
narrow absorption line with emissions at both sides. We can see that the central absorption increases and progresses towards the red side with phase, and the central reversal emission appears in Figures $1 \mathrm{c}$ and 1d. In totality just after the second contact the emission at the red-side disappears, as shown in Figure 1d.

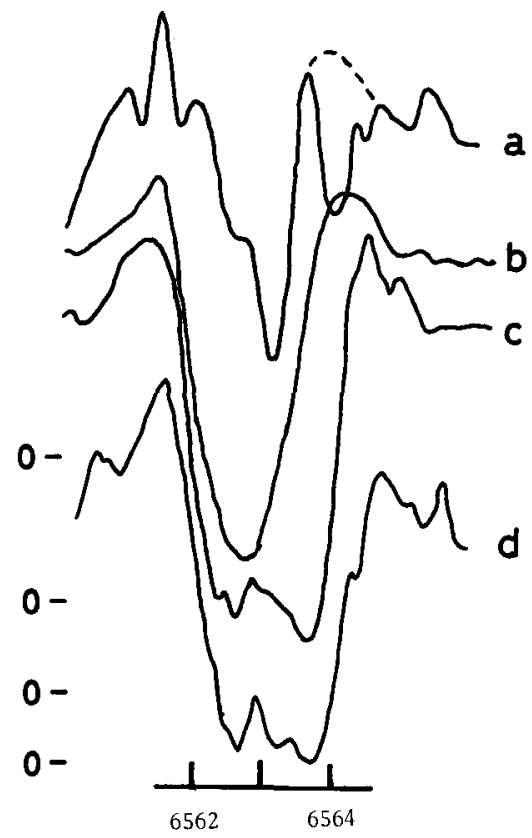

Fig. 1. Profiles of Epsilon Aurigae around the $\mathrm{H} \alpha$ line. Plate No. and the date observed are: (a) $\mathrm{C} 10-3636$, 21 November, 1978, (b) C10-3970, 10 December, 1981, (c) C10-4082, 9 December, 1982, and (d) C10-4104, 27 January, 1983. There is a small flaw on plate (a). A corrected profile is drawn by a dashed curve in profile (a). The abscissas are arbitrary scale.

The disappearance of the red-side emission in totality was reported by Guinan (1983) and Boehm and Ferluga (1983). In the last eclipse, Wright and Kushwaha (1957) found the same phenomenon.

Figure 2 shows intensities at three phases against outside eclipse as functions of wavelength around $H \alpha$. The decrease of continuum radiation has been estimated from the V-magnitude light curve of Ingvarsson (1983) at each phase. We can see from Figure 2 that (1) strong absorption of $\mathrm{H} \alpha$ line has appeared with radial velocity of $-5 \mathrm{~km} \mathrm{~s}^{-1}$ even at 10 December, 1981, at seven months before the first contact, 29 July, 1982 (Gyldenkerne, 1970), and the absorption gradually increases with phase, (2) at ingress and totality, absorption has been rapidly increasing at red side with radial velocity of $40 \mathrm{~km} \mathrm{~s}^{-1}$, and (3) the eclipse at the $\mathrm{H} \alpha$ line becomes almost complete by the two absorption components, although half the continuum radiation is appearing during totality. 


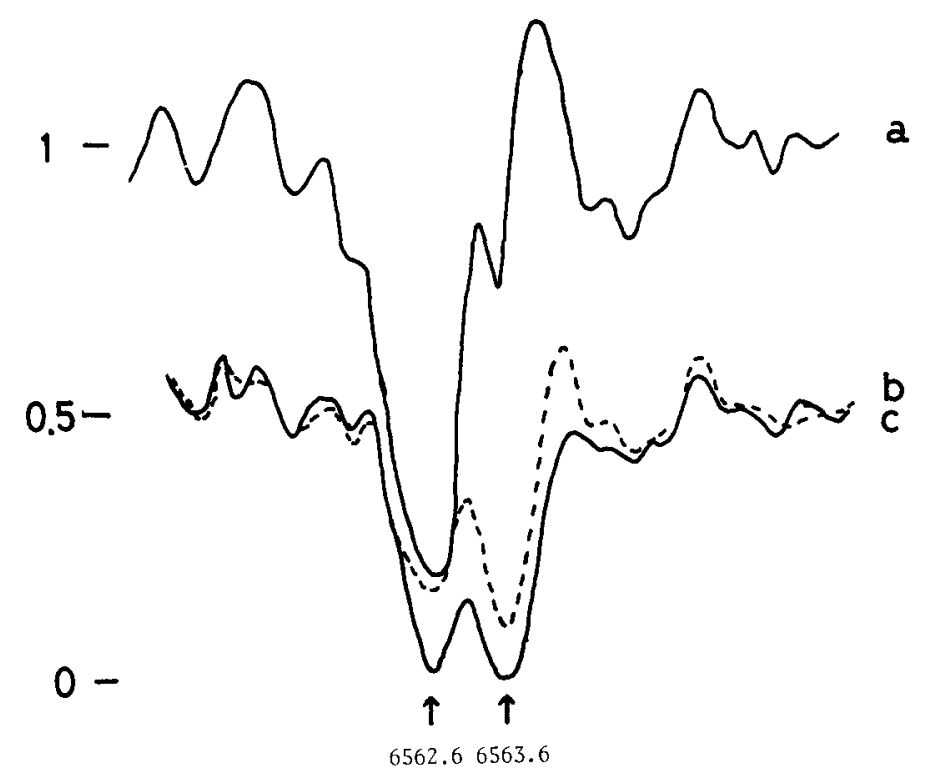

Fig. 2. Intensities around the $\mathrm{H} \alpha$ line at eclipsing phases relative to intensity of the plate $\mathrm{C} 10-3636$ obtained outside eclipse. Plate No. and the date observed are: (a) C10-3970, 10 December, 1981, (b) C10-4082, 9 December, 1982, and (c) C10-4102, 27 January, 1983.

\section{Radial Velocities of Absorption Lines}

Figure 3 shows radial velocity curves of absorption lines of neutral hydrogen and metals around the second contact. The center of gravity of the binary system moves $-2.5 \mathrm{~km} \mathrm{~s}^{-1}$. Our measurements have been made for seven plates of blue and ultraviolet regions with a dispersion of $4.1 \AA$ per $\mathrm{mm}$.

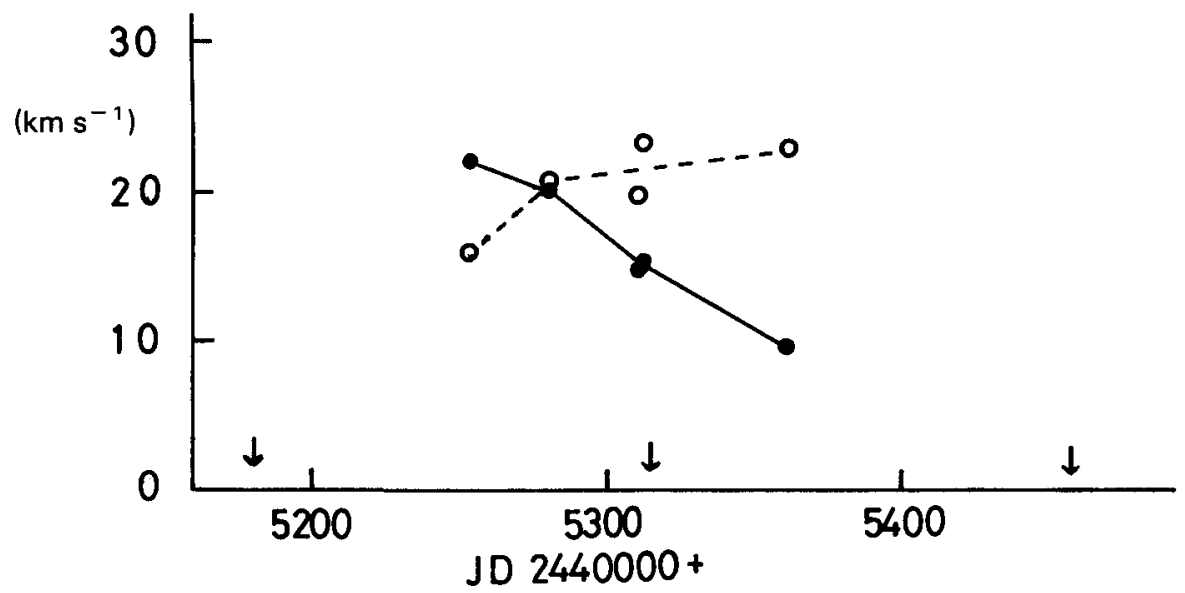

Fig. 3. Radial velocities of neutral hydrogen, denoted by open circles, and neutral metals, denoted by filled circles. Three arrows show first and second contacts and mid-eclipse predicted by Gyldenkerne (1970). 
The Balmer line velocities slightly increase with phase. The velocity increase corresponds to the development of the red-side absorption of the $\mathrm{H} \alpha$ line with phase mentioned above. On the other hand, the radial velocities of neutral metals decrease almost linearly with phase towards zero velocity at mid-eclipse. Profiles of the neutral metals are asymmetrical with steeper gradient at the red side and the intensities scarcely change during the phases shown in Figure 3 in spite of the large variations of the radial velocities.

Such a separation of the radial velocity curves between neutral hydrogen and neutral metals has also appeared around the second and third contacts of the last eclipse (Wright, 1970).

\section{Model of the Secondary Component}

We may consider from the results obtained in the previous sections that the secondary consists of at least three parts in structure:

(1) Neutral metals are confined in a ring which is rotating with $20 \mathrm{~km} \mathrm{~s}^{-1}$ or more, because of the linearly decreasing radial velocity curve and of the almost constant intensities of the absorption lines.

(2) Neutral hydrogen with radial velocities of $40 \mathrm{~km} \mathrm{~s}^{-1}$ distributes in the ring of metals and also inside the ring and it eclipses all over the photosphere of the primary. The observations of Wright and Kushwaha (1957) for the last eclipse show that neutral hydrogen layer seems to be rotating with velocities increasing towards the center.

(3) A low-density neutral hydrogen envelope extends at least twice the radius of the ring of metals. The radial velocity of the envelope is almost equal to that of the binary system.

\section{References}

Boehm, C. and Ferluga, S.: 1983, Comm. 27, IAU Inf. Bull. Var. Stars, No. 2326.

Gaposchkin, S.: 1954, Publ. Astron. Soc. Pacific 66, 112.

Guinan, E.: 1983, Epsilon Aur Campaign Newsletter 7, 7.

Gyldenkerne, K.: 1970, Vistas Astron. 12, 199.

Hack, M.: 1959, Astrophys. J. 129, 291.

Huang, S.-S.: 1965, Astrophys. J. 141, 976.

Ingvarsson, S. I.: 1983, Epsilon Aur Campaign Newsletter 6, 8.

Kopal, Z.: 1954, The Observatory 74, 14.

Kopal, Z.: 1971, Astrophys. Space Sci. 10, 332.

Kuiper, G. P., Struve, O., and Strömgren, B.: 1937, Astrophys. J. 86, 570.

Wilson, R. E.: 1971, Astrophys. J. 170, 529.

Wright, K. O.: 1970, Vistas Astron. 12, 147.

Wright, K. O. and Kushwaha, R. S.: 1957, Comm. Coll. Intern. Astrophys. Liège 8, 421. 